\title{
Article \\ Female Reproductive Factors and the Risk of Bronchiectasis: A Nationwide Population-Based Longitudinal Study
}

\author{
Bumhee Yang ${ }^{1,+} \oplus^{\mathbb{D}}$, Dong-Hwa Lee ${ }^{2,+} \mathbb{D}^{\mathbb{D}}$, Kyungdo Han ${ }^{3}$, Hayoung Choi ${ }^{4} \mathbb{D}$, Hyung Koo Kang ${ }^{5} \mathbb{D}$, \\ Dong Wook Shin ${ }^{6,7}$ and Hyun Lee ${ }^{8, *(D)}$
}

1 Division of Pulmonary and Critical Care Medicine, Department of Internal Medicine, Chungbuk National University Hospital, Chungbuk National University College of Medicine, Cheongju 28644, Korea; ybhworld0415@gmail.com

2 Division of Endocrinology and Metabolism, Department of Internal Medicine, Chungbuk National University Hospital, Chungbuk National University College of Medicine, Cheongju 28644, Korea; roroko@hanmail.net

3 Department of Statistics and Actuarial Science, Soongsil University, Seoul 06978, Korea; hkd917@naver.com

4 Division of Pulmonary, Allergy, and Critical Care Medicine, Department of Internal Medicine, Hallym University Kangnam Sacred Heart Hospital, Hallym University College of Medicine, Seoul 07441, Korea; hychoimd@gmail.com

5 Division of Pulmonary and Critical Care Medicine, Department of Internal Medicine, Inje University Ilsan Paik Hospital, Inje University College of Medicine, Goyang 10380, Korea; inspirit26@gmail.com

6 Department of Family Medicine \& Supportive Care Center, Samsung Medical Center, Sungkyunkwan University School of Medicine, Seoul 06351, Korea; dwshin.md@gmail.com

7 Department of Clinical Research Design and Evaluation/Digital Health, Samsung Advanced Institute of Health Science and Technology (SAIHST), Sungkyunkwan University, Seoul 06351, Korea

8 Division of Pulmonary Medicine and Allergy, Department of Internal Medicine, Hanyang University College of Medicine, Seoul 04763, Korea

* Correspondence: namuhanayeyo@hanyang.ac.kr or namuhanayeyo@naver.com

+ These authors contributed equally to the work, as co-first authors.

Citation: Yang, B.; Lee, D.-H.; Han, K.; Choi, H.; Kang, H.K.; Shin, D.W.; Lee, H. Female Reproductive Factors and the Risk of Bronchiectasis: A Nationwide Population-Based Longitudinal Study. Biomedicines 2022, 10, 303. https://doi.org/ $10.3390 /$ biomedicines 10020303

Academic Editors: Célia F. Rodrigues and Natália Cruz-Martins

Received: 6 January 2022

Accepted: 25 January 2022

Published: 28 January 2022

Publisher's Note: MDPI stays neutral with regard to jurisdictional claims in published maps and institutional affiliations.

Copyright: (C) 2022 by the authors. Licensee MDPI, Basel, Switzerland. This article is an open access article distributed under the terms and conditions of the Creative Commons Attribution (CC BY) license (https:// creativecommons.org/licenses/by/ $4.0 /)$.
Abstract: Although the oestrogen level is thought to be involved in the occurrence of bronchiectasis, limited data are available on the relationship between female reproductive factors and the risk of bronchiectasis. We performed a population-based retrospective cohort study of 959,523 premenopausal women and 1,362,401 postmenopausal women without a previous history of bronchiectasis who participated in a health screening exam in 2009 in South Korea. In premenopausal women, compared with a later age at menarche ( $\geq 16$ years), an earlier menarche ( $<12$ years) was associated with a reduced risk of bronchiectasis with an adjusted hazard ratio (aHR) $(95 \%$ confidence interval (CI)) of $0.74(0.67-0.81)$. However, there were no significant associations between other reproductive factors (breastfeeding, parity, or oral contraceptive use) and the risk of bronchiectasis. In postmenopausal women, the risk of bronchiectasis (aHR $(95 \% \mathrm{CI})$ ) was lower in those with an earlier menarche $(0.79(0.72-0.87)$ for $<12$ years vs. $\geq 16$ years $)$, a later menopause $(0.90(0.84-0.96) \geq 55$ years vs. $<40$ years), and a longer reproductive period $(0.90(0.86-0.94)$ for $\geq 40$ years vs. $<30$ years). There was no significant relationship between parity and the risk of bronchiectasis. Although breastfeeding $<1$ year $($ aHR $(95 \%$ CI $)=0.92(0.87-0.97)$ for $<0.5$ years and $0.93(0.88-0.97)$ for $0.5-1$ years $)$ and oral contraceptive use $<1$ year $(0.97$ (0.94-0.99)) reduced the risk of bronchiectasis, hormone replacement therapy $\geq 5$ years increased the risk of bronchiectasis $(1.24$ (1.18-1.30)). Female reproductive factors are risk factors for developing bronchiectasis, showing a higher risk associated with shorter endogenous oestrogen exposure regardless of the menopausal status.

Keywords: female; sex hormone; reproductive period; menarche; menopause; bronchiectasis

\section{Introduction}

In adults, women generally are more vulnerable than men to the development of airway diseases [1-4] and have a higher susceptibility to the damaging effects of noxious 
particles (smoking, air pollution, etc.) in the respiratory system [3,5,6]. Female sex hormones including 17ß-estradiol have been suggested to contribute to this sex disparity and many previous studies have evaluated the associations between female sex hormones and the development of chronic airway diseases [1,2,7-14].

Despite several conflicting results, those previous study findings have provided novel insights into the link between female sex hormones and many chronic respiratory diseases [1,8-14]. In non-cystic fibrosis bronchiectasis (hereafter bronchiectasis), a few previous studies found a sex disparity in prevalence; the prevalence of bronchiectasis in women was affected by their reproductive span [2,4,15-23]. Although those results suggested a close relationship between the lifetime oestrogen exposure and the development of bronchiectasis, no study has considered comprehensive female reproductive factors (age at menarche, age at menopause, parity, breastfeeding, oral contraceptive (OC) use, and hormone replacement therapy (HRT)) [2,4,15-21].

In this study, we evaluated the connections between female reproductive factors and the development of bronchiectasis. In our analyses, we used a population-based longitudinal cohort and several female reproductive factors.

\section{Materials and Methods}

\subsection{Study Population and Design}

We gathered our data from the Korean National Health Insurance Service (NHIS) database [24]. Korea has a single-payer universal health system; the NHIS maintains the claims data on all reimbursed inpatient and outpatient visits, procedures, and prescriptions including data from annual (all Koreans 40 years or older and all employees regardless of age) or biennial (workers in jobs requiring physical labour) health screening exams provided free of charge by the Ministry of Health and Welfare [25,26]. The health screening exams include the National Cancer Screening Program, which screens all Korean women aged 40 or older biennially for breast cancer [27]. During that screening program, all participants are asked to complete a self-administered questionnaire on reproductive factors (age at menarche, age at menopause, parity, breastfeeding, OC use, and HRT).

This study initially included 3,109,506 females older than 40 years who received a health screening exam in 2009 (index year). After excluding 314,529 participants with an uncertain menopause status, our study population contained 1,069,475 premenopausal and $1,725,502$ postmenopausal participants. Among the 1,069,475 premenopausal participants, we identified 959,523 eligible participants after excluding those: (1) missing information for at least one variable $(n=99,644)$; (2) diagnosed with cystic fibrosis or congenital bronchiectasis $(n=85)$; (3) diagnosed with bronchiectasis before the enrolment period $(n=8044)$; (4) diagnosed with bronchiectasis within one year after enrolment $(n=1734)$; or (5) who died within one year of enrolment $(n=445)$. Among the $1,725,502$ postmenopausal participants, we identified 1,362,401 eligible participants after excluding those: (1) missing information for at least one variable $(n=320,845)$; (2) diagnosed with cystic fibrosis or congenital bronchiectasis $(n=115)$; (3) diagnosed with bronchiectasis before the enrolment period $(n=31,556)$; (4) diagnosed with bronchiectasis within one year after enrolment ( $n=6689)$; or (5) who died within one year of enrolment $(n=3896)$ (Figure 1). The participants were tracked from the index year until the date of a bronchiectasis diagnosis, death, or 31 December 2018.

Our study protocol was approved by the Institutional Review Board of Chungbuk National University Hospital (No. 2021-08-016). The requirement for informed consent was waived because the NHIS database uses an anonymous patient identification system.

\subsection{Exposure}

The main exposures were age at menarche in the premenopausal women and reproductive duration in the postmenopausal women. The data on the age at menarche and the age at menopause were obtained from a self-administered questionnaire. The age at menarche was categorised as $<12$ years, $12-14$ years $(\geq 12,<14), 14-16$ years $(\geq 14,<16)$, 
and $\geq 16$ years. The age at menopause was categorised as $<40$ years, $40-45$ years $(\geq 40,<45)$, $45-50$ years $(\geq 45,<50), 50-55$ years $(\geq 50,<55)$ and $\geq 55$ years. The reproductive period was calculated as the interval between the age at menarche and the age at menopause [28]. The reproductive period was categorised as $<30$ years, 30-35 years $(\geq 30,<35)$, 35-40 years $(\geq 35,<40)$, and $\geq 40$ years.

Other exposures included parity, breastfeeding, and OC use in all women and HRT in postmenopausal women. The data on parity, lifetime breastfeeding history, OC use, and HRT were also obtained from a self-administered questionnaire. The parity was categorised as none, one child, or multiparous ( $\geq 2$ children). The lifetime breastfeeding history was categorised as none, $<0.5$ years, $0.5-1(\geq 0.5,<1)$ years, and $\geq 1$ year. The duration of HRT was categorised as none, $<2$ years, $2-5(\geq 2,<5)$ years, and $\geq 5$ years. The duration of OC use was categorised as none, $<1$ year, and $\geq 1$ year [28].

Women aged over 40 years who participated in a health screening exam between 1 January 2009 and 31 December 2012 $(n=3,109,506)$

Missing information $(n=99,644)$

Cystic fibrosis or congenital bronchiectasis $(n=85)$ Bronchiectasis diagnosed before the enrolment period $(\mathrm{n}=8044)$

Bronchiectasis diagnosed within one year after enrolment $(n=1734)$

Died within one year after enrolment $(n=445)$

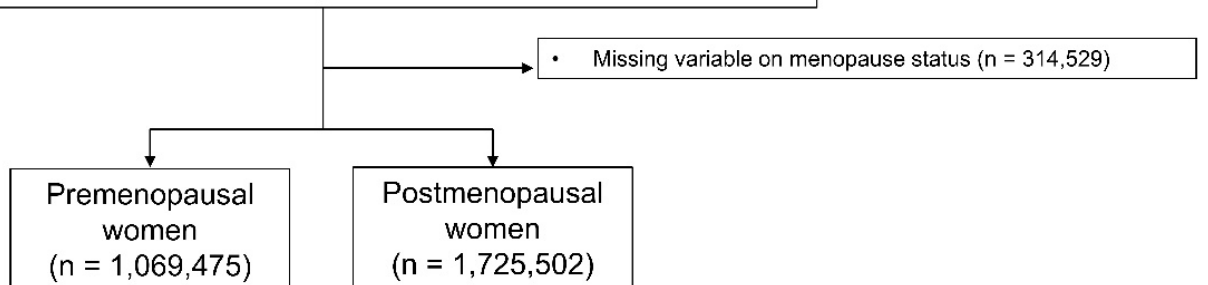

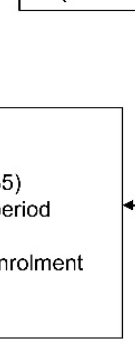
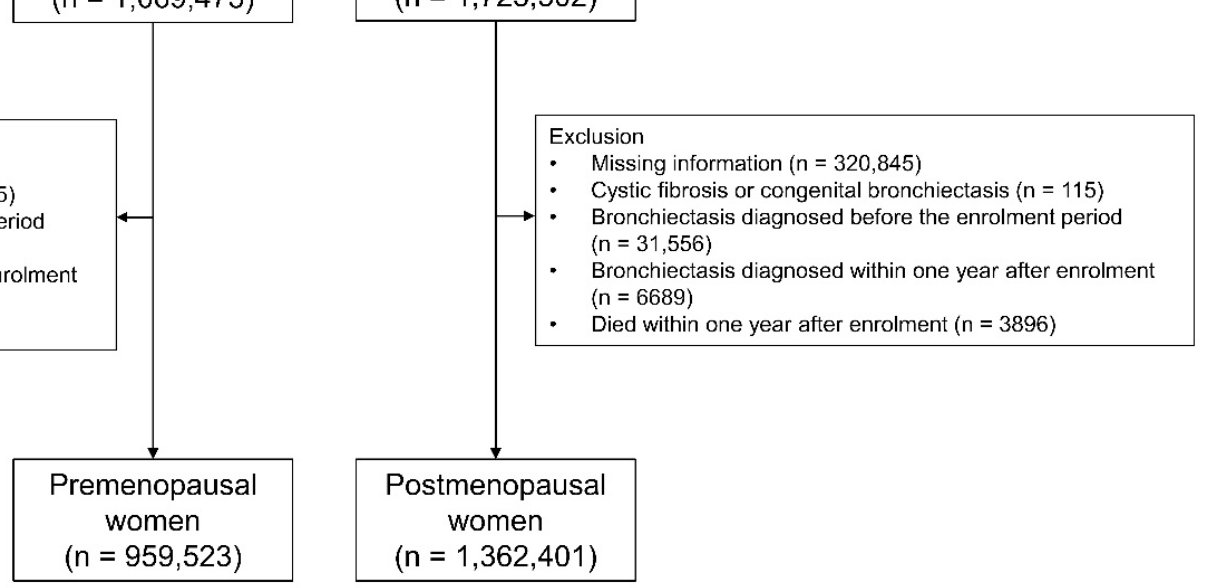

Figure 1. Flow chart of the study population.

\subsection{Outcome}

The main study outcome was incidence of bronchiectasis. Bronchiectasis was defined as claims under the International Statistical Classification of Diseases and Related Health Problems, 10th revision (ICD-10) diagnosis code J47 (bronchiectasis) without a concomitant diagnosis of cystic fibrosis (E84), as used in our previous studies [29-33].

\subsection{Covariates}

The body mass index (BMI) was calculated as the weight of a participant in kilograms divided by the square of height in meters. The smoking status was classified as nonsmoker, ex-smoker, and current smoker. Regular exercise was defined as moderate physical activity for more than 30 min on more than 5 days of the past week [34]. Comorbidities (hypertension, diabetes mellitus, dyslipidaemia, strokes, cardiovascular disease, asthma or chronic obstructive pulmonary disease (COPD), and tuberculosis) were collected using ICD-10 codes in the medical records of the participants [29-35]. The household income was categorised into quartiles based on the insurance premium levels (in Korea, insurance premiums are determined by the income level) with those covered by medical aid (poorest $3 \%$ ) being merged into the lowest income quartile [28,34]. 


\subsection{Statistical Analysis}

The continuous variables were presented as mean \pm standard deviation (SD) and the categorical variables were presented as a number and percentage. The incidence rate of bronchiectasis was expressed as the number of events per 1000 person/years. Cox proportional hazard regression analyses were conducted to evaluate the associations between the various reproductive factors and the incidence of bronchiectasis. The baseline demographics, metabolic comorbidities that might interact with the female reproductive factors (diabetes mellitus and dyslipidaemia), and pulmonary comorbidities that might affect the development of bronchiectasis (e.g., asthma [36,37], COPD [38], and tuberculosis [39]) were considered in the multivariable analyses. For premenopausal women, the multivariable model was adjusted for age, BMI, smoking history, regular exercise, income, age at menarche, parity, duration of breastfeeding, duration of OC use, and comorbidities (diabetes mellitus, dyslipidaemia, asthma or COPD, and tuberculosis). For postmenopausal women, the multivariable model was adjusted for age, BMI, smoking history, regular exercise, income, reproductive period, parity, duration of breastfeeding, duration of OC use, duration of HRT, and comorbidities (diabetes mellitus, dyslipidaemia, asthma or COPD, and tuberculosis). All statistical analyses were performed using SAS version 9.4 (SAS Institute Inc., Cary, NC, USA).

\section{Results}

\subsection{Baseline Characteristics}

The baseline characteristics of the study population are described in Table 1. During a mean follow-up of 8.3 years, 15,069 premenopausal women were diagnosed with bronchiectasis for an incidence rate of 190.2 cases per 100,000 person/years. Compared with women who did not develop bronchiectasis, those who developed bronchiectasis had a higher mean age ( 46.9 vs. 45.1 years), a lower BMI $\left(22.9\right.$ vs. $\left.23.2 \mathrm{~kg} / \mathrm{m}^{2}\right)$, a higher proportion of current and ex-smokers (5.2\% vs. $5.0 \%$ ), and more comorbidities: dyslipidaemia (12.7 vs. $11.4 \%)$, cardiovascular disease ( $1.8 \%$ vs. $0.9 \%)$, asthma or COPD $(14.4 \%$ vs. $8.3 \%)$, and tuberculosis $(10.9 \%$ vs. $4.1 \%)$ ( $p<0.01$ for all variables). Among the reproductive factors, premenopausal women who developed bronchiectasis were more likely than those who did not have menarche at an older age ( 15.4 vs. 15.1 years), who were nulliparous ( $14.0 \%$ vs. $13.3 \%)$, and who had breastfed for $0.5-1$ year ( $35.8 \%$ vs. $31.3 \%)(p<0.01$ for all variables). OC use did not differ between those two groups $(p=0.18)$.

During a mean follow-up of 8.1 years, 51,669 postmenopausal women were diagnosed with bronchiectasis for an incidence rate of 468.7 cases per 100,000 person/years. Compared with women who did not develop bronchiectasis, those who developed bronchiectasis were more likely to be older (63.1 vs. 61.6 years), current or ex-smokers $(4.1 \%$ vs. $3.7 \%)$, have a lower BMI (23.8 vs. $\left.24.2 \mathrm{~kg} / \mathrm{m}^{2}\right)$, and have more comorbidities (cardiovascular disease ( $7.2 \%$ vs. $5.6 \%)$, asthma or COPD (23.1\% vs. $13.0 \%)$, and tuberculosis $(12.1 \%$ vs. $6.2 \%)$. However, women who developed bronchiectasis did not exercise regularly compared with those who did exercise regularly $(82.6 \%$ vs. $81.6 \%)(p<0.01$ for all variables). With respect to reproductive factors, postmenopausal women who developed bronchiectasis were more likely than those who did not have an older age at menarche (16.6 vs. 16.5 years), who were multiparous $(92.3 \%$ vs. $91.8 \%)$, and who had breastfed for $\geq 1$ year $(73.1 \%$ vs. $70.5 \%)(p<0.01$ for all variables). The rate of participants who received HRT $\geq 5$ years was significantly higher among those who developed bronchiectasis than among those who did $\operatorname{not}(3.3 \%$ vs. $2.8 \%, p<0.01)$.

\subsection{Reproductive Factors and the Risk of Bronchiectasis in Premenopausal Women}

As shown in Table 2, the multivariable Cox regression analyses showed that an earlier menarche correlated with a lower risk of bronchiectasis (adjusted hazard ratio (aHR) (95\% CI) for age at menarche: $0.74(0.67-0.81)$ for < 12 years; $0.78(0.75-0.82)$ for $12-14$ years; 0.89 (0.86-0.93) for 14-16 years) in premenopausal women. Other reproductive factors (parity, duration of breastfeeding, and OC use) were not associated with a risk of bronchiectasis. 
Similarly, the incidence probability (\%) of bronchiectasis differed significantly among the premenopausal women with menarche at age $\geq 16$ years, those at $14-16$ years, and those at $<14$ years $(p$ for log-rank test $<0.01)$.

Table 1. Baseline characteristics of the study population.

\begin{tabular}{|c|c|c|c|c|c|c|}
\hline & \multicolumn{3}{|c|}{ Premenopausal Women } & \multicolumn{3}{|c|}{ Postmenopausal Women } \\
\hline & $\begin{array}{l}\text { Subjects Who } \\
\text { Did Not } \\
\text { Develop } \\
\text { Bronchiectasis } \\
(n=944,454)\end{array}$ & $\begin{array}{c}\text { Subjects Who } \\
\text { Did Develop } \\
\text { Bronchiectasis } \\
(n=15,069)\end{array}$ & $p$-Value & $\begin{array}{l}\text { Subjects Who } \\
\text { Did Not } \\
\text { Develop } \\
\text { Bronchiectasis } \\
(n=1,310,732)\end{array}$ & $\begin{array}{l}\text { Subjects Who } \\
\text { Did Develop } \\
\text { Bronchiectasis } \\
(n=51,669)\end{array}$ & $p$-Value \\
\hline Age (years) & $45.1 \pm 4.3$ & $46.9 \pm 4.9$ & $<0.01$ & $61.6 \pm 8.3$ & $63.1 \pm 8.2$ & $<0.01$ \\
\hline BMI $\left(\mathrm{kg} / \mathrm{m}^{2}\right)$ & $23.2 \pm 3.1$ & $22.9 \pm 3.1$ & $<0.01$ & $24.2 \pm 3.2$ & $23.8 \pm 3.3$ & $<0.01$ \\
\hline Smoking history & & & $<0.01$ & & & $<0.01$ \\
\hline Non-smoker & $897,230(95.0)$ & $14,294(94.8)$ & & $1,262,129(96.3)$ & $49,536(95.9)$ & \\
\hline Ex-smoker & $15,132(1.6)$ & $213(1.5)$ & & $13,705(1.1)$ & $600(1.2)$ & \\
\hline Current smoker & $32,092(3.4)$ & $562(3.7)$ & & $34,898(2.6)$ & $1533(2.9)$ & \\
\hline Regular physical activity & & & 0.10 & & & $<0.01$ \\
\hline No & $780,458(82.6)$ & $12,529(83.2)$ & & $1,069,822(81.6)$ & $42,643(82.6)$ & \\
\hline Yes & $163,996(17.4)$ & $2540(16.8)$ & & $240,910(18.4)$ & $9026(17.4)$ & \\
\hline Income (quartile) & & & $<0.01$ & & & $<0.01$ \\
\hline Q1 (lowest) & $243,033(25.7)$ & $3913(25.9)$ & & $297,366(22.7)$ & $11,324(21.9)$ & \\
\hline Q2 & $191,698(20.3)$ & $3177(21.1)$ & & $243,680(18.6)$ & $9305(18.0)$ & \\
\hline Q3 & $209,064(22.1)$ & 3405 (22.6) & & $324,561(24.8)$ & $12,718(24.6)$ & \\
\hline Q4 (highest) & $300,659(31.9)$ & $4574(30.4)$ & & $445,125(33.9)$ & $18,322(35.5)$ & \\
\hline Age at menarche (years) & $15.1 \pm 1.7$ & $15.4 \pm 1.7$ & $<0.01$ & $16.5 \pm 1.8$ & $16.6 \pm 1.9$ & $<0.01$ \\
\hline Age at menopause (years) & & & & $50.0 \pm 4.1$ & $49.9 \pm 4.1$ & $<0.01$ \\
\hline Reproductive period (years) & & & & $33.5 \pm 4.4$ & $33.3 \pm 4.5$ & $<0.01$ \\
\hline Parity & & & $<0.01$ & & & $<0.01$ \\
\hline Nulliparous & $125,964(13.3)$ & $2110(14.0)$ & & $30,511(2.3)$ & $1217(2.4)$ & \\
\hline One child & $780,039(82.0)$ & $12,401(82.3)$ & & $77,231(5.9)$ & $2738(5.3)$ & \\
\hline Multiparous & $38,451(4.7)$ & $558(3.7)$ & & $1,202,990(91.8)$ & $47,714(92.3)$ & \\
\hline $\begin{array}{l}\text { Duration of breastfeeding } \\
\text { (years) }\end{array}$ & & & $<0.01$ & & & $<0.01$ \\
\hline None & $230,001(24.3)$ & $3350(22.2)$ & & $83,450(6.4)$ & $3175(6.1)$ & \\
\hline$<0.5$ & $248,072(26.2)$ & $3880(25.8)$ & & $81,805(6.3)$ & $2829(5.5)$ & \\
\hline $0.5-1$ & $295,720(31.3)$ & $5393(35.8)$ & & $220,694(16.8)$ & $7910(15.3)$ & \\
\hline$\geq 1$ & $170,661(18.0)$ & $2446(16.2)$ & & $924,783(70.5)$ & $37,755(73.1)$ & \\
\hline Duration of OC use (years) & & & 0.18 & & & $<0.01$ \\
\hline None & $819,633(86.7)$ & $13,011(86.3)$ & & $1,103,598(84.2)$ & $43,783(84.7)$ & \\
\hline$<1$ & $91,645(9.7)$ & $1492(9.9)$ & & $123,987(9.5)$ & $4652(9.0)$ & \\
\hline$\geq 1$ & $33,176(3.5)$ & $566(3.8)$ & & $83,147(6.3)$ & $3234(6.3)$ & \\
\hline Duration of HRT (years) & & & & & & $<0.01$ \\
\hline None & & & & $1,104,212(84.2)$ & $42,837(82.9)$ & \\
\hline$<2$ & & & & $120,365(9.3)$ & $4985(9.7)$ & \\
\hline $2-5$ & & & & $49,025(3.7)$ & $2095(4.1)$ & \\
\hline$\geq 5$ & & & & $37,130(2.8)$ & $1752(3.3)$ & \\
\hline \multicolumn{7}{|l|}{ Comorbidities } \\
\hline Diabetes mellitus & $34,637(3.6)$ & $560(3.7)$ & 0.75 & $176,001(13.4)$ & $6544(12.6)$ & $<0.01$ \\
\hline Dyslipidaemia & $108,512(11.4)$ & 1917 (12.7) & $<0.01$ & $452,441(34.5)$ & $17,336(33.5)$ & $<0.01$ \\
\hline Cardiovascular disease & $4669(0.9)$ & $145(1.8)$ & $<0.01$ & $50,617(5.6)$ & $2597(7.2)$ & $<0.01$ \\
\hline Asthma or COPD & $78,514(8.3)$ & $2176(14.4)$ & $<0.01$ & $171,479(13.0)$ & $11,949(23.1)$ & $<0.01$ \\
\hline Tuberculosis & $38,681(4.1)$ & $1644(10.9)$ & $<0.01$ & $82,289(6.2)$ & $6250(12.1)$ & $<0.01$ \\
\hline
\end{tabular}


Table 2. HR and 95\% CI for the associations between reproductive factors and the risk of bronchiectasis among premenopausal women.

\begin{tabular}{|c|c|c|c|c|c|c|}
\hline & \multirow{2}{*}{ Total $(n)$} & \multirow{2}{*}{$\begin{array}{c}\text { Bronchiectasis } \\
(n)\end{array}$} & \multirow{2}{*}{$\begin{array}{c}\text { Follow-Up } \\
\text { Duration (PY) }\end{array}$} & \multirow{2}{*}{ IR (/1000 PY) } & \multicolumn{2}{|c|}{ HR $(95 \%$ CI) } \\
\hline & & & & & Univariable & Multivariable \\
\hline Age (years) & & & & & $1.07(1.07,1.08)$ & $1.07(1.07,1.08)$ \\
\hline BMI $\left(\mathrm{kg} / \mathrm{m}^{2}\right)$ & & & & & $0.97(0.96,0.97)$ & $0.95(0.95,0.96)$ \\
\hline \multicolumn{7}{|l|}{ Smoking history } \\
\hline Non-smoker & 911,524 & 14,294 & $7,528,360.8$ & 1.89 & Reference & Reference \\
\hline Ex-smoker & 15,345 & 213 & $126,443.2$ & 1.68 & $0.88(0.77,1.01)$ & $0.98(0.85,1.12)$ \\
\hline Current smoker & 32,654 & 562 & $268,561.0$ & 2.09 & $1.10(1.01,1.19)$ & $1.14(1.04,1.24)$ \\
\hline \multicolumn{7}{|c|}{ Regular physical activity } \\
\hline No & 792,987 & 12,529 & $6,545,086.2$ & 1.91 & Reference & Reference \\
\hline Yes & 166,536 & 2540 & $1,378,278.9$ & 1.84 & $0.96(0.92,1.01)$ & $0.95(0.91,1.00)$ \\
\hline \multicolumn{7}{|c|}{ Age at menarche (years) } \\
\hline$<12$ & 42,696 & 505 & $352,080.1$ & 1.43 & $0.56(0.51,0.61)$ & $0.74(0.67,0.81)$ \\
\hline $12-14$ & 301,146 & 3792 & $2,486,061.3$ & 1.52 & $0.60(0.57,0.62)$ & $0.78(0.75,0.82)$ \\
\hline $14-16$ & 439,342 & 7059 & $3,627,513.6$ & 1.94 & $0.76(0.73,0.79)$ & $0.89(0.86,0.93)$ \\
\hline$\geq 16$ & 176,339 & 3713 & $1,457,709.0$ & 2.54 & Reference & Reference \\
\hline \multicolumn{7}{|l|}{ Parity } \\
\hline Nulliparous & 39,009 & 558 & $320,903.4$ & 1.73 & Reference & Reference \\
\hline One child & 128,074 & 2110 & $1,056,351.2$ & 1.99 & $1.14(1.04,1.26)$ & $1.03(0.93,1.14)$ \\
\hline Multiparous & 792,440 & 12,401 & $6,546,110.7$ & 1.89 & $1.08(1.00,1.18)$ & $1.01(0.92,1.11)$ \\
\hline \multicolumn{7}{|c|}{$\begin{array}{l}\text { Duration of } \\
\text { breastfeeding (years) }\end{array}$} \\
\hline None & 173,107 & 2446 & $1,427,337.2$ & 1.71 & Reference & Reference \\
\hline$<0.5$ & 233,351 & 3350 & $1,924,498.6$ & 1.74 & $1.01(0.96,1.07)$ & $0.97(0.92,1.03)$ \\
\hline $0.5-1$ & 251,952 & 3880 & $2,081,080.5$ & 1.86 & $1.08(1.03,1.14)$ & $0.99(0.93,1.04)$ \\
\hline$\geq 1$ & 301,113 & 5393 & $2,490,448.7$ & 2.16 & $1.26(1.20,1.32)$ & $1.05(0.99,1.11)$ \\
\hline \multicolumn{7}{|l|}{$\begin{array}{l}\text { Duration of OC use } \\
\text { (years) }\end{array}$} \\
\hline None & 832,644 & 13,011 & $6,875,528.4$ & 1.89 & Reference & Reference \\
\hline$<1$ & 93,137 & 1492 & $769,027.2$ & 1.94 & $1.02(0.97,1.08)$ & $1.04(0.98,1.09)$ \\
\hline \multirow[t]{2}{*}{$\geq 1$} & 33,742 & 566 & $278,809.5$ & 2.03 & $1.07(0.98,1.16)$ & $1.02(0.93,1.11)$ \\
\hline & $\begin{array}{l}\text { Data are } \\
\text { regular es } \\
\text { (diabetes } \\
\text { hazard ra } \\
\text { pulmona }\end{array}$ & $\begin{array}{l}\text { esented as } n \text { or } \\
\text { cise, income, age } \\
\text { ellitus, dyslipid } \\
\text {; CI: confidence } \\
\text { disease. }\end{array}$ & $\begin{array}{l}95 \% \text { CI). The mu } \\
\text { nenarche, parity, } \\
\text { ia, asthma /COP } \\
\text { rval; BMI: body }\end{array}$ & $\begin{array}{l}\text { ariable model } \\
\text { ation of breastfe } \\
\text { and tuberculosi } \\
\text { s index; OC: or }\end{array}$ & $\begin{array}{l}\text { s adjusted for age, } \\
\text { ing, duration of OC } \\
\text { PY: person/years; I } \\
\text { contraceptive; COP }\end{array}$ & $\begin{array}{l}\text { MI, smoking histor } \\
\text { e, and comorbiditi } \\
\text { incidence rate; } \mathrm{H} \\
\text { chronic obstructiv }\end{array}$ \\
\hline
\end{tabular}

\subsection{Reproductive Factors and the Risk of Bronchiectasis in Postmenopausal Women}

As shown in Table 3, the univariable Cox regression analyses showed that an earlier menarche, a later menopause, and a longer reproductive period reflecting a longer exposure to female sex hormones decreased the risk of bronchiectasis in a dose-dependent manner. As those three variables were highly correlated, only the reproductive period was included in the multivariable analyses. After adjusting for the potential confounders, a longer reproductive period correlated with a lower risk of bronchiectasis (aHR (95\% CI) for the reproductive period: 0.97 (0.95-0.99) for 30-35 years; $0.94(0.91-0.96)$ for 35-40 years; 0.90 (0.86-0.94) for $\geq 40$ years). Similarly, Figure 2B shows that the cumulative incidence probability (\%) of bronchiectasis differed significantly among the postmenopausal women with a reproductive period $<30$ years, those with a period of 30-35 years, and those with a period $\geq 35$ years ( $p$ for log-rank test $<0.01$ ). 
Table 3. HR and 95\% CI for the associations between reproductive factors and the risk of bronchiectasis among postmenopausal women.

\begin{tabular}{|c|c|c|c|c|c|c|}
\hline & \multirow{2}{*}{ Total $(n)$} & \multirow{2}{*}{$\begin{array}{c}\text { Bronchiectasis } \\
\text { (n) }\end{array}$} & \multirow{2}{*}{$\begin{array}{c}\text { Follow-Up } \\
\text { Duration (PY) }\end{array}$} & \multirow{2}{*}{ IR (/1000 PY) } & \multicolumn{2}{|c|}{ HR (95\% CI) } \\
\hline & & & & & Univariable & Multivariable \\
\hline Age (years) & & & & & $1.02(1.02,1.03)$ & $1.02(1.02,1.03)$ \\
\hline BMI $\left(\mathrm{kg} / \mathrm{m}^{2}\right)$ & & & & & $0.96(0.95,0.96)$ & $0.96(0.96,0.97)$ \\
\hline \multicolumn{7}{|l|}{ Smoking history } \\
\hline Non-smoker & $1,311,665$ & 49,536 & $10,623,789.8$ & 4.66 & Reference & Reference \\
\hline Ex-smoker & 14,305 & 600 & $113,888.1$ & 5.26 & $1.13(1.04,1.22)$ & $1.13(1.04,1.23)$ \\
\hline Current smoker & 36,431 & 1533 & $287,390.0$ & 5.33 & $1.14(1.08,1.20)$ & $1.10(1.05,1.16)$ \\
\hline \multicolumn{7}{|c|}{ Regular physical activity } \\
\hline No & $1,112,465$ & 42,643 & $8,985,784.4$ & 4.74 & Reference & Reference \\
\hline Yes & 249,936 & 9026 & $2,039,283.6$ & 4.42 & $0.93(0.91,0.95)$ & $0.96(0.94,0.98)$ \\
\hline \multicolumn{7}{|c|}{ Age at menarche (years) } \\
\hline$<12$ & 12,954 & 418 & $105,648.2$ & 3.96 & $0.79(0.72-0.87)$ & \\
\hline $12-14$ & 164,560 & 5586 & $1,339,752.9$ & 4.17 & $0.83(0.81-0.86)$ & \\
\hline $14-16$ & 524,836 & 18,996 & $4,255,170.5$ & 4.46 & $0.89(0.88-0.91)$ & \\
\hline$\geq 16$ & 660,051 & 26,669 & $5,324,496.4$ & 5.01 & Reference & \\
\hline \multicolumn{7}{|c|}{ Age at menopause (years) } \\
\hline$<40$ & 24,290 & 1003 & $194,175.6$ & 5.17 & Reference & \\
\hline $40-45$ & 80,042 & 3225 & $641,612.4$ & 5.03 & $0.97(0.91,1.05)$ & \\
\hline $45-50$ & 371,284 & 14,078 & $3,004,544.3$ & 4.69 & $0.91(0.85,0.97)$ & \\
\hline $50-55$ & 740,042 & 27,841 & $5,994,369.0$ & 4.64 & $0.90(0.85,0.96)$ & \\
\hline$\geq 55$ & 146,743 & 5522 & $1,190,366.4$ & 4.64 & $0.90(0.84,0.96)$ & \\
\hline \multicolumn{7}{|c|}{ Reproductive period (years) } \\
\hline$<30$ & 192,647 & 8018 & $1,543,989.8$ & 5.19 & Reference & Reference \\
\hline $30-35$ & 568,223 & 22,028 & $4,590,126.7$ & 4.80 & $0.95(0.90,0.95)$ & $0.97(0.95,0.99)$ \\
\hline $35-40$ & 513,918 & 18,479 & $4,179,829.6$ & 4.42 & $0.85(0.83,0.87)$ & $0.94(0.91,0.96)$ \\
\hline$\geq 40$ & 87,613 & 3144 & $711,121.8$ & 4.42 & $0.85(0.82,0.89)$ & $0.90(0.86,0.94)$ \\
\hline \multicolumn{7}{|l|}{ Parity } \\
\hline Nulliparous & 31,728 & 1217 & $257,028.2$ & 4.73 & Reference & Reference \\
\hline One child & 79,969 & 2738 & $650,986.6$ & 4.21 & $0.89(0.83,0.95)$ & $0.95(0.88,1.02)$ \\
\hline Multiparous & $1,250,704$ & 47,714 & $10,117,053.2$ & 4.72 & $0.99(0.94,1.05)$ & $0.98(0.92,1.04)$ \\
\hline \multicolumn{7}{|c|}{$\begin{array}{l}\text { Duration of breastfeeding } \\
\text { (years) }\end{array}$} \\
\hline None & 86,625 & 3175 & $703,992.0$ & 4.51 & Reference & Reference \\
\hline$<0.5$ & 84,634 & 2829 & $690,832.9$ & 4.10 & $0.91(0.86,0.96)$ & $0.92(0.87,0.97)$ \\
\hline $0.5-1$ & 228,604 & 7910 & $1,860,046.3$ & 4.25 & $0.94(0.91,0.98)$ & $0.93(0.88,0.97)$ \\
\hline$\geq 1$ & 962,538 & 37,755 & $7,770,196.7$ & 4.86 & $1.08(1.04,1.12)$ & $0.98(0.94,1.02)$ \\
\hline \multicolumn{7}{|c|}{ Duration of OC use (years) } \\
\hline None & $1,147,381$ & 43,783 & $9,274,646.3$ & 4.72 & Reference & Reference \\
\hline$<1$ & 128,639 & 4652 & $1,048,247.7$ & 4.44 & $0.94(0.91,0.97)$ & $0.97(0.94,0.99)$ \\
\hline$\geq 1$ & 86,381 & 3234 & $702,174.0$ & 4.61 & $0.98(0.94,1.01)$ & $0.98(0.94,1.01)$ \\
\hline \multicolumn{7}{|c|}{ Duration of HRT (years) } \\
\hline None & $1,147,049$ & 42,837 & $9,265,189.4$ & 4.62 & Reference & Reference \\
\hline$<2$ & 125,350 & 4985 & $1,025,341.5$ & 4.86 & $1.05(1.02,1.08)$ & $1.16(1.12,1.19)$ \\
\hline $2-5$ & 51,120 & 2095 & $417,429.4$ & 5.02 & $1.09(1.04,1.13)$ & $1.17(1.12,1.23)$ \\
\hline$\geq 5$ & 38,882 & 1752 & $317,107.6$ & 5.52 & $1.20(1.14,1.25)$ & $1.24(1.18,1.30)$ \\
\hline
\end{tabular}

Data are presented as $\mathrm{n}$ or HR $(95 \% \mathrm{CI})$. The multivariable model was adjusted for age, BMI, smoking history, regular exercise, income, reproductive period, parity, duration of breastfeeding, duration of OC use, duration of HRT, and comorbidities (hypertension, diabetes mellitus, dyslipidaemia, asthma/COPD, and tuberculosis). PY: person/years; IR: incidence rate; HR: hazard ratio; CI: confidence interval; BMI: body mass index; OC: oral contraceptive; HRT: hormone replacement therapy; COPD: chronic obstructive pulmonary disease.

Regarding other reproductive factors and the risk of bronchiectasis, breastfeeding duration $<1$ year $($ aHR $(95 \% \mathrm{CI})=0.92(0.87-0.97)$ for $<0.5$ years and $0.93(0.88-0.97)$ for $0.5-1$ years) compared with no breastfeeding and OC use $<1$ year (adjusted HR $(95 \% \mathrm{CI})=0.97(0.94-0.99))$ compared with no use were associated with a decreased risk of bronchiectasis. In contrast, the duration of HRT dose-dependently increased the risk of bronchiectasis (aHR (95\% CI) for HRT duration: 1.16 (1.12-1.19) for < 2 years, $1.17(1.12-1.23)$ for 2-5 years, 1.24 (1.18-1.30) for $\geq 5$ years). 
(A)

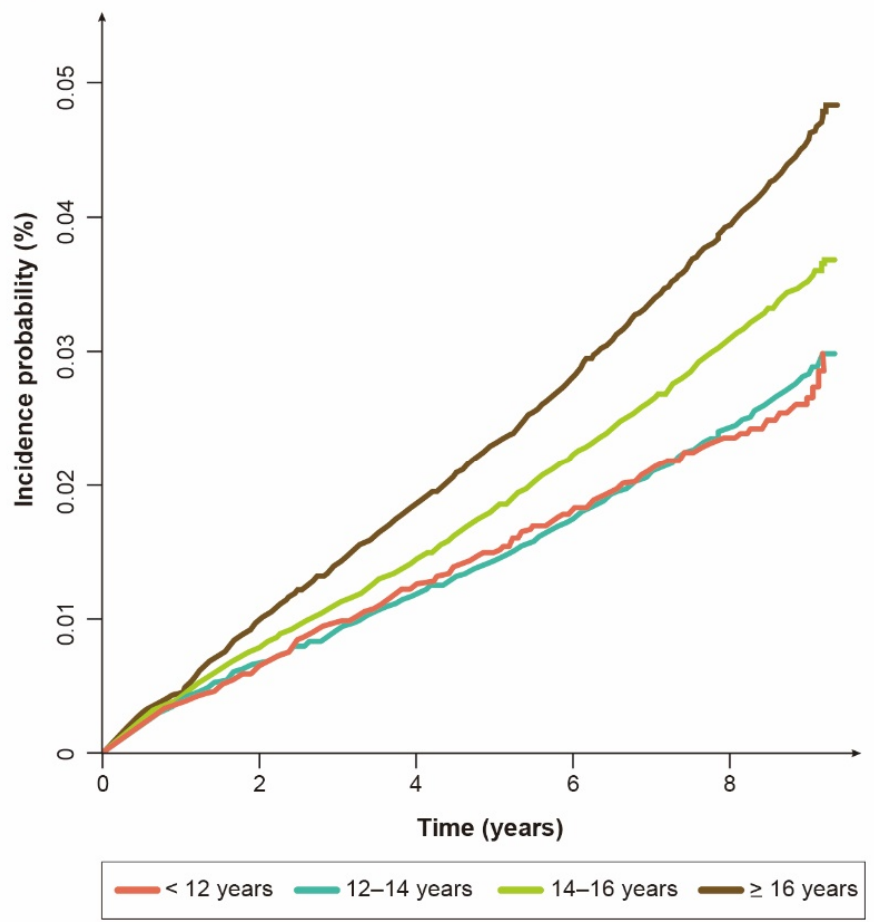

(B)

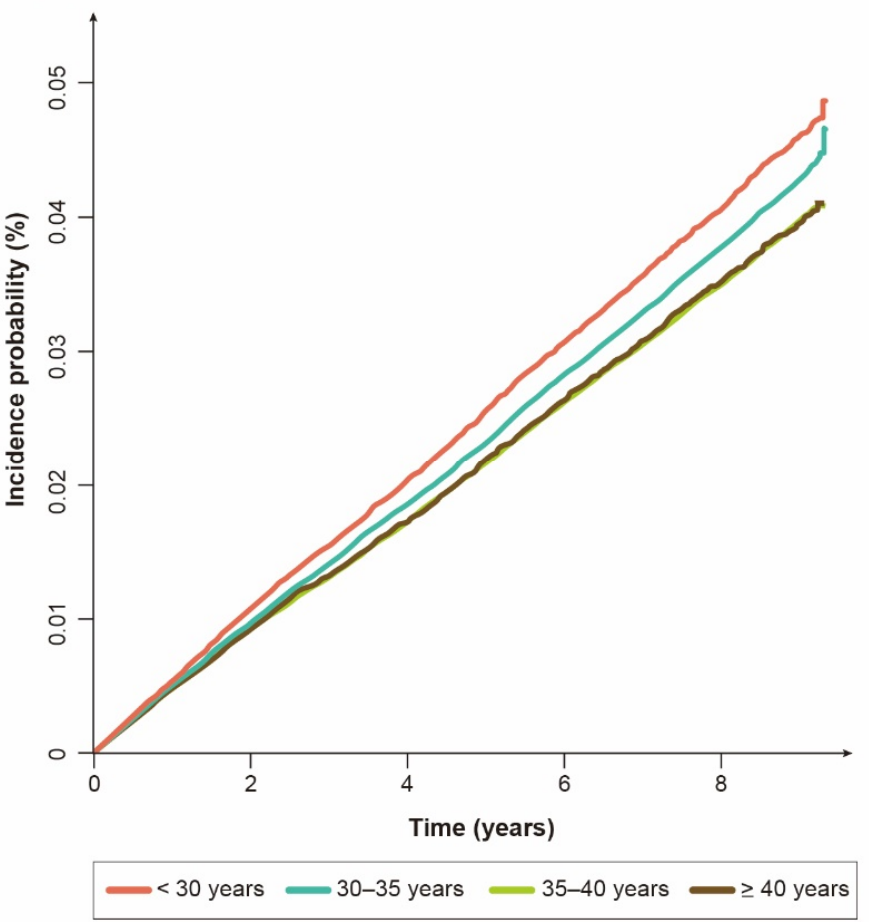

Figure 2. Cumulative incidence probability (\%) of bronchiectasis: (A) premenopausal women according to age at menarche; (B) postmenopausal women according to reproductive period.

\section{Discussion}

In this population-based longitudinal cohort study, we investigated the associations between female reproductive factors and the risk of bronchiectasis. Even after adjusting for potential confounders, a shorter lifetime exposure to endogenous female sex hormones (a later menarche in premenopausal women and a later menarche, earlier menopause, and shorter reproductive period in postmenopausal women) correlated with an increased incidence of bronchiectasis.

As the lifetime duration of endogenous oestrogen exposure varies among women and oestrogen is involved in inflammatory signalling in the airways, it can be postulated that lifetime oestrogen exposure affects the development of lung diseases. Accordingly, previous studies evaluated the effects of female reproductive factors on inflammatory lung diseases such as asthma and COPD [1,8-14]. However, the association between lifetime oestrogen exposure and the development of bronchiectasis has not been elucidated well. Most previous studies provided indirect evidence such as sex disparity in the prevalence of bronchiectasis [2,4,15-23]. Others supported that view by showing the prevalence of bronchiectasis in women affected by their reproductive span $[19,23]$. However, those previous studies used a cross-sectional methodology and did not consider other reproductive factors $[2,4,15-23]$. Thus, the major strengths of our study were that we used a population-based longitudinal cohort and considered the complexity of various reproductive parameters (parity, breastfeeding, OC use, and HRT) to assess the relationship between lifetime oestrogen exposure and the development of bronchiectasis.

Another important advantage of our study was that we considered comorbidity profiles that might affect the development of bronchiectasis in the analyses of the association of female sex hormones with bronchiectasis. Previous studies have shown that airway diseases such as asthma [36,37], COPD [38], and pulmonary tuberculosis [39] are major aetiologies of bronchiectasis. For example, Crimi et al. showed a link between asthma and bronchiectasis by demonstrating that a type 2 inflammation could provide a causative role in the development of bronchiectasis [36,37]. Additionally, a recent longitudinal study showed that bronchiectasis could develop in subjects with COPD who had bronchial 
infections; post-tuberculosis bronchiectasis is known to be one of the leading aetiologies of bronchiectasis worldwide [38,39]. Furthermore, as subjects with those comorbidities are more likely to have a low BMI, which can affect female reproductive factors [40-43], there might be skewed outcomes if these comorbidities were not adjusted. Thus, the comprehensive adjustment of these comorbidities highlights the reliability of our analyses.

The most important finding of our study was that we are, to the best of our knowledge, the first to demonstrate the protective effect of endogenous female sex hormones (probably oestrogen effects because oestrogen exposure is longer than progesterone exposure during the female reproductive cycle) against the development of bronchiectasis. Supporting our results, a few previous studies that evaluated the prevalence of bronchiectasis by sex and age found that the prevalence in women increased abruptly around the beginning of the menopause $[19,23]$. Previous basic research using a murine model of asthma also suggested that oestrogen might have a protective role against bronchiectasis [44-46]. In those studies, oestrogen was shown to have an anti-inflammatory role in the airway by reducing leukocyte recruitment, mucus production, and the secretion of pro-inflammatory cytokines [44-46]. Thus, the clinical relevance of our study was on the finding that the evaluation of bronchiectasis might be helpful in women with a short reproductive duration and unexplained respiratory symptoms.

Unexpectedly, we found that HRT was associated with an increased risk of bronchiectasis in postmenopausal women. Although the reasons for that were unclear, we suggest that our current results were most likely a reflection of reverse causality. In other words, women with more severe symptoms due to a more substantial deficiency in endogenous sex hormones (more vulnerable to the development of bronchiectasis) might have had received more HRT than those whose milder symptoms represented a tolerable endogenous sex hormone deficiency, as shown in other studies $[47,48]$.

However, there were other possibilities for the positive relationship between HRT and the risk of bronchiectasis in postmenopausal women. First, a timing hypothesis was suggested that the timing of oestrogen replacement treatment could determine whether it has beneficial or harmful effects on other diseases $[47,49]$. In line with that view, oestrogen was shown to enhance mucus synthesis in human bronchial epithelial cells as well as dehydrate airway surfaces and reduce cilia beat frequency [50], which could contribute to the progression of bronchiectasis. Synthetic medroxyprogesterone acetate, a major component of HRT, could be second possible explanation. A previous study showed that progesterone inhibited the cilia beat frequency in the airway epithelium, which could inhibit mucociliary clearance [50]. In Korea, most drugs prescribed as HRT in 2010 were progesterogenic (53\% used an oestrogen-progesterone combination and $40 \%$ used Tibolone, which has a weak estrogenic, progestogenic, and androgenic activity [51]) [52]. Unfortunately, our database lacked accurate data on the type of hormone formulation used and the age at which HRT was initiated. Thus, well-designed future studies are needed to confirm the association between HRT and the risk of bronchiectasis.

Although our study provided the important finding that female sex hormones and the development of bronchiectasis were linked, it had a few limitations. First, the diagnosis of bronchiectasis was made by physicians, which is a major limitation of all claims data-based studies. Thus, there might have been an over- or under-estimation of bronchiectasis. Second, information regarding the reproductive factors was obtained from a health questionnaire so recall bias could not be ruled out. Third, the relationship between the reproductive factors that can lead to relatively short-term changes in life endogenous oestrogen exposure (breastfeeding duration or OC use) and the risk of bronchiectasis in postmenopausal women did not show consistent results; breastfeeding $<1$ year was associated with a reduced risk of bronchiectasis whereas breastfeeding $\geq 1$ year did not show a significant relationship with the risk of bronchiectasis. OC use did not show any relationship with the risk of bronchiectasis in premenopausal women but OC use $<1$ year reduced the risk of bronchiectasis in postmenopausal women. Although these inconsistent results might have been influenced by the relatively short-term exposure of those factors, we could not provide 
a plausible explanation for this phenomenon. Fourth, because this study was conducted in an exclusively Korean population, generalisability might be limited. Fifth, a vitamin $\mathrm{D}$ deficiency is an important factor associated with bronchiectasis [53] but we could not include vitamin D levels in the analyses as these data were not available in our database.

\section{Conclusions}

In conclusion, our results suggest that endogenous female sex hormones might have a protective role against the development of bronchiectasis.

Author Contributions: Literature search: all authors; study design: B.Y., D.-H.L., K.H. and H.L.; data analysis: K.H. and H.C.; data interpretation: all authors; writing: B.Y., D.-H.L., H.K.K., D.W.S. and H.L.; tables and figures: B.Y., D.-H.L., K.H. and H.L. All authors have read and agreed to the published version of the manuscript.

Funding: This work was supported by grants from the National Research Foundation (NRF) of Korea (2020R1A5A2017476). This work was also supported by the National Research Foundation of Korea (NRF), grant funded by the Ministry of Science, Information and Communications Technologies (NRF-2020R1F1A1070468 and 2021M3E5D1A01015176), and the Korea Medical Device Development Fund, grant funded by the Korean government (the Ministry of Science and ICT, the Ministry of Trade, Industry and Energy, the Ministry of Health \& Welfare, and the Ministry of Food and Drug Safety) (Project Number: 1711138447, KMDF_PR_20200901_0214).

Institutional Review Board Statement: This study protocol was approved by the Institutional Review Board of Chungbuk National University Hospital (No. 2021-08-016).

Informed Consent Statement: Not applicable.

Data Availability Statement: Not applicable.

Conflicts of Interest: The authors declare no conflict of interest.

\section{References}

1. Leynaert, B.; Sunyer, J.; Garcia-Esteban, R.; Svanes, C.; Jarvis, D.; Cerveri, I.; Dratva, J.; Gislason, T.; Heinrich, J.; Janson, C.; et al. Gender differences in prevalence, diagnosis and incidence of allergic and non-allergic asthma: A population-based cohort. Thorax 2012, 67, 625-631. [CrossRef] [PubMed]

2. Raghavan, D.; Jain, R. Increasing awareness of sex differences in airway diseases. Respirology 2016, 21, 449-459. [CrossRef] [PubMed]

3. Sørheim, I.-C.; Johannessen, A.; Gulsvik, A.; Bakke, P.S.; Silverman, E.K.; DeMeo, D.L. Gender differences in COPD: Are women more susceptible to smoking effects than men? Thorax 2010, 65, 480-485. [CrossRef] [PubMed]

4. Vidaillac, C.; Yong, V.F.L.; Jaggi, T.K.; Soh, M.-M.; Chotirmall, S.H. Gender differences in bronchiectasis: A real issue? Breathe 2018, 14, 108-121. [CrossRef]

5. Gan, W.Q.; Man, S.F.; Postma, D.S.; Camp, P.; Sin, D.D. Female smokers beyond the perimenopausal period are at increased risk of chronic obstructive pulmonary disease: A systematic review and meta-analysis. Respir. Res. 2006, 7, 52. [CrossRef]

6. Clougherty, J.E. A growing role for gender analysis in air pollution epidemiology. Environ. Health Perspect. 2010, 118, 167-176. [CrossRef]

7. Scioscia, G.; Carpagnano, G.E.; Lacedonia, D.; Soccio, P.; Quarato, C.M.I.; Trabace, L.; Fuso, P.; Barbaro, M.P.F. The Role of Airways 17beta-Estradiol as a Biomarker of Severity in Postmenopausal Asthma: A Pilot Study. J. Clin. Med. 2020, 9, 2037. [CrossRef]

8. Barr, R.G.; Wentowski, C.C.; Grodstein, F.; Somers, S.C.; Stampfer, M.J.; Schwartz, J.; Speizer, F.E.; Camargo, C.A., Jr. Prospective Study of Postmenopausal Hormone Use and Newly Diagnosed Asthma and Chronic Obstructive Pulmonary Disease. Arch. Intern. Med. 2004, 164, 379-386. [CrossRef]

9. Troisi, R.J.; Speizer, F.E.; Willett, W.C.; Trichopoulos, D.; Rosner, B. Menopause, postmenopausal estrogen preparations, and the risk of adult-onset asthma. A prospective cohort study. Am. J. Respir. Crit. Care Med. 1995, 152, 1183-1188. [CrossRef]

10. Romieu, I.; Fabre, A.; Fournier, A.; Kauffmann, F.; Varraso, R.; Mesrine, S.; Leynaert, B.; Clavel-Chapelon, F. Postmenopausal hormone therapy and asthma onset in the E3N cohort. Thorax 2010, 65, 292-297. [CrossRef]

11. Triebner, K.; Johannessen, A.; Puggini, L.; Benediktsdóttir, B.; Bertelsen, R.J.; Bifulco, E.; Dharmage, S.C.; Dratva, J.; Franklin, K.A.; Gíslason, T.; et al. Menopause as a predictor of new-onset asthma: A longitudinal Northern European population study. J. Allergy Clin. Immunol. 2016, 137, 50-57.e6. [CrossRef] [PubMed]

12. Shah, S.A.; Tibble, H.; Pillinger, R.; McLean, S.; Ryan, D.; Critchley, H.; Price, D.; Hawrylowicz, C.M.; Simpson, C.R.; Soyiri, I.N.; et al. Hormone replacement therapy and asthma onset in menopausal women: National cohort study. J. Allergy Clin. Immunol. 2021, 147, 1662-1670. [CrossRef] [PubMed] 
13. Tang, R.; Fraser, A.; Magnus, M.C. Female reproductive history in relation to chronic obstructive pulmonary disease and lung function in UK biobank: A prospective population-based cohort study. BMJ Open 2019, 9, e030318. [CrossRef] [PubMed]

14. van der Plaat, D.A.; Pereira, M.; Pesce, G.; Potts, J.F.; Amaral, A.F.S.; Dharmage, S.C.; Garcia-Aymerich, J.M.; Thompson, J.R.; Gómez-Real, F.; Jarvis, D.L.; et al. Age at menopause and lung function: A Mendelian randomisation study. Eur. Respir. J. 2019, 54, 1802421. [CrossRef] [PubMed]

15. Lee, H.; Choi, H.; Chalmers, J.D.; Dhar, R.; Nguyen, T.Q.; Visser, S.K.; Morgan, L.C.; Oh, Y.-M. Characteristics of bronchiectasis in Korea: First data from the Korean Multicentre Bronchiectasis Audit and Research Collaboration registry and comparison with other international registries. Respirology 2021, 26, 619-621. [CrossRef] [PubMed]

16. Martinez-García, M.A.; Villa, C.; Dobarganes, Y.; Girón, R.; Maíz, L.; García-Clemente, M.; Sibila, O.; Golpe, R.; Rodríguez, J.; Barreiro, E.; et al. RIBRON: The Spanish online bronchiectasis registry. Characterization of the first 1912 patients. Arch. Bronconeumol. Engl. Ed. 2021, 57, 28-35. [CrossRef]

17. Snell, N.; Gibson, J.; Jarrold, I.; Quint, J.K. Epidemiology of bronchiectasis in the UK: Findings from the British lung foundation's 'Respiratory health of the nation' project. Respir. Med. 2019, 158, 21-23. [CrossRef]

18. Huang, H.-Y.; Chung, F.-T.; Lo, C.-Y.; Lin, H.-C.; Huang, Y.-T.; Yeh, C.-H.; Lin, C.-W.; Huang, Y.-C.; Wang, C.-H. Etiology and characteristics of patients with bronchiectasis in Taiwan: A cohort study from 2002 to 2016. BMC Pulm. Med. 2020, 20, 45. [CrossRef]

19. Aliberti, S.; Sotgiu, G.; Lapi, F.; Gramegna, A.; Cricelli, C.; Blasi, F. Prevalence and incidence of bronchiectasis in Italy. BMC Pulm. Med. 2020, 20, 15. [CrossRef]

20. Visser, S.K.; Bye, P.T.P.; Fox, G.J.; Burr, L.D.; Chang, A.B.; Holmes-Liew, C.-L.; King, P.; Middleton, P.G.; Maguire, G.P.; Smith D.; et al. Australian adults with bronchiectasis: The first report from the Australian Bronchiectasis Registry. Respir. Med. 2019, 155, 97-103. [CrossRef]

21. Ringshausen, F.C.; Rademacher, J.; Pink, I.; de Roux, A.; Hickstein, L.; Ploner, T.; Welte, T.; Diel, R. Increasing bronchiectasis prevalence in Germany, 2009-2017: A population-based cohort study. Eur. Respir. J. 2019, 54, 1900499. [CrossRef] [PubMed]

22. Aksamit, T.R.; O’Donnell, A.E.; Barker, A.; Olivier, K.N.; Winthrop, K.L.; Daniels, M.L.A.; Johnson, M.; Eden, E.; Griffith, D.; Knowles, M.; et al. Adult Patients With Bronchiectasis: A First Look at the US Bronchiectasis Research Registry. Chest 2017, 151, 982-992. [CrossRef]

23. Weycker, D.; Hansen, G.L.; Seifer, F.D. Prevalence and incidence of noncystic fibrosis bronchiectasis among US adults in 2013 Chronic Respir. Dis. 2017, 14, 377-384. [CrossRef] [PubMed]

24. Song, S.O.; Jung, C.H.; Song, Y.D.; Park, C.-Y.; Kwon, H.-S.; Cha, B.-S.; Park, J.-Y.; Lee, K.-U.; Ko, K.S.; Lee, B.-W. Background and data configuration process of a nationwide population-based study using the korean national health insurance system. Diabetes Metab. J. 2014, 38, 395-403. [CrossRef] [PubMed]

25. National Health Insurence Service. National Health Examination Statistical Yearbook 2014; NHIS: Seoul, Korea, 2014.

26. Lee, Y.-H.; Han, K.; Ko, S.-H.; Ko, K.S.; Lee, K.-U.; Taskforce Team of Diabetes Fact Sheet of the Korean Diabetes Association. Data Analytic Process of a Nationwide Population-Based Study Using National Health Information Database Established by National Health Insurance Service. Diabetes Metab. J. 2016, 40, 79-82. [CrossRef]

27. Yoo, K.-Y. Cancer Control Activities in the Republic of Korea. Jpn. J. Clin. Oncol. 2008, 38, 327-333. [CrossRef]

28. Yoo, J.E.; Shin, D.W.; Han, K.; Kim, D.; Yoon, J.W.; Lee, D.-Y. Association of Female Reproductive Factors With Incidence of Fracture Among Postmenopausal Women in Korea. JAMA Netw. Open 2021, 4, e2030405. [CrossRef]

29. Choi, H.; Yang, B.; Nam, H.; Kyoung, D.S.; Sim, Y.S.; Park, H.Y.; Lee, J.S.; Lee, S.W.; Oh, Y.M.; Ra, S.W.; et al. Population-based prevalence of bronchiectasis and associated comorbidities in South Korea. Eur. Respir. J. 2019, 54, 1900194. [CrossRef]

30. Yang, B.; Ryu, J.; Kim, T.; Jo, Y.S.; Kim, Y.; Park, H.Y.; Kang, Y.A.; Lee, S.J.; Lee, O.-J.; Moon, J.-Y.; et al. Impact of Bronchiectasis on Incident Nontuberculous Mycobacterial Pulmonary Disease: A 10-Year National Cohort Study. Chest 2021, 159, $1807-1811$. [CrossRef]

31. Choi, H.; Lee, H.; Ryu, J.; Chung, S.J.; Park, D.W.; Sohn, J.W.; Yoon, H.J.; Kim, S.-H. Bronchiectasis and increased mortality in patients with corticosteroid-dependent severe asthma: A nationwide population study. Ther. Adv. Respir. Dis. 2020, 14, 1753466620963030. [CrossRef]

32. Yang, B.; Han, K.; Kim, S.H.; Lee, D.-H.; Park, S.H.; Yoo, J.E.; Shin, D.W.; Choi, H.; Lee, H. Being Underweight Increases the Risk of Non-Cystic Fibrosis Bronchiectasis in the Young Population: A Nationwide Population-Based Study. Nutrients 2021, $13,3206$. [CrossRef] [PubMed]

33. Choi, H.; Yang, B.; Kim, Y.J.; Sin, S.; Jo, Y.S.; Kim, Y.; Park, H.Y.; Ra, S.W.; Oh, Y.-M.; Chung, S.J.; et al. Increased mortality in patients with non cystic fibrosis bronchiectasis with respiratory comorbidities. Sci. Rep. 2021, 11, 7126. [CrossRef] [PubMed]

34. Yoo, J.E.; Kim, D.; Han, K.; Rhee, S.Y.; Shin, D.W.; Lee, H. Diabetes Status and Association with Risk of Tuberculosis among Korean Adults. JAMA Netw. Open 2021, 4, e2126099. [CrossRef] [PubMed]

35. Lee, H.; Ryu, J.; Nam, E.; Chung, S.J.; Yeo, Y.; Park, D.W.; Park, T.S.; Moon, J.-Y.; Kim, T.-H.; Sohn, J.W.; et al. Increased mortality in patients with corticosteroid-dependent asthma: A nationwide population-based study. Eur. Respir. J. 2019, 54, 1900804. [CrossRef] [PubMed]

36. Crimi, C.; Ferri, S.; Campisi, R.; Crimi, N. The Link between Asthma and Bronchiectasis: State of the Art. Respir. Int. Rev. Thorac. Dis. 2020, 99, 463-476. [CrossRef] [PubMed] 
37. Crimi, C.; Campisi, R.; Nolasco, S.; Ferri, S.; Cacopardo, G.; Impellizzeri, P.; Pistorio, M.P.; Fagone, E.; Pelaia, C.; Heffler, E.; et al. Type 2-High Severe Asthma with and without Bronchiectasis: A Prospective Observational Multicentre Study. J. Asthma Allergy 2021, 14, 1441-1452. [CrossRef]

38. Martínez-García, M.; de la Rosa-Carrillo, D.; Soler-Cataluña, J.J.; Catalan-Serra, P.; Ballester, M.; Roca Vanaclocha, Y.; Agramunt, M.; Ballestin, J.; Garcia-Ortega, A.; Oscullo, G.; et al. Bronchial Infection and Temporal Evolution of Bronchiectasis in Patients With Chronic Obstructive Pulmonary Disease. Clin. Infect. Dis. 2021, 72, 403-410. [CrossRef]

39. Chandrasekaran, R.; Mac Aogáin, M.; Chalmers, J.D.; Elborn, S.J.; Chotirmall, S.H. Geographic variation in the aetiology, epidemiology and microbiology of bronchiectasis. BMC Pulm. Med. 2018, 18, 83. [CrossRef]

40. Zhu, D.; Chung, H.F.; Pandeya, N.; Dobson, A.J.; Kuh, D.; Crawford, S.L.; Gold, E.B.; Avis, N.E.; Giles, G.G.; Bruinsma, F.; et al. Body mass index and age at natural menopause: An international pooled analysis of 11 prospective studies. Eur. J. Epidemiol. 2018, 33, 699-710. [CrossRef]

41. Casha, A.R.; Scarci, M. The link between tuberculosis and body mass index. J. Thorac. Dis. 2017, 9, E301-E303. [CrossRef]

42. McDonald, M.N.; Wouters, E.F.M.; Rutten, E.; Casaburi, R.; Rennard, S.I.; Lomas, D.A.; Bamman, M.; Celli, B.; Agusti, A.; Tal-Singer, R.; et al. It's more than low BMI: Prevalence of cachexia and associated mortality in COPD. Respir. Res. 2019, 20, 100. [CrossRef]

43. Sio, Y.Y.; Chew, F.T. Risk factors of asthma in the Asian population: A systematic review and meta-analysis. J. Physiol. Anthropol. 2021, 40, 22. [CrossRef] [PubMed]

44. Cheng, C.; Wu, H.; Wang, M.; Wang, L.; Zou, H.; Li, S.; Liu, R. Estrogen ameliorates allergic airway inflammation by regulating activation of NLRP3 in mice. Biosci. Rep. 2019, 39, BSR20181117. [CrossRef] [PubMed]

45. Dimitropoulou, C.; Drakopanagiotakis, F.; Chatterjee, A.; Snead, C.; Catravas, J.D. Estrogen replacement therapy prevents airway dysfunction in a murine model of allergen-induced asthma. Lung 2009, 187, 116-127. [CrossRef] [PubMed]

46. Riffo-Vasquez, Y.; Ligeiro de Oliveira, A.P.; Page, C.P.; Spina, D.; Tavares-de-Lima, W. Role of sex hormones in allergic inflammation in mice. Clin. Exp. Allergy 2007, 37, 459-470. [CrossRef] [PubMed]

47. Lee, H.; Choi, H.; Sim, Y.S.; Park, S.; Kim, W.J.; Yoo, K.H.; Lee, S.J.; Kim, T.-H.; Yang, B.; Jeong, I.; et al. KMBARC registry: Protocol for a multicentre observational cohort study on non-cystic fibrosis bronchiectasis in Korea. BMJ Open 2020, 10, e034090. [CrossRef]

48. Eun, Y.; Yoo, J.E.; Han, K.D.; Kim, D.H.; Lee, J.; Lee, D.Y.; Lee, D.H.; Kim, H.; Shin, D.W. OP0113 female reproductive factors and risk of joint replacement arthroplasty of knee and hip due to osteoarthritis in postmenopausal women: A nationwide cohort study of 1.36 million women. Ann. Rheum. Dis. 2021, 80, 62-63. [CrossRef]

49. Bassuk, S.S.; Manson, J.E. The timing hypothesis: Do coronary risks of menopausal hormone therapy vary by age or time since menopause onset? Metabolism 2016, 65, 794-803. [CrossRef]

50. Jain, R.; Ray, J.M.; Pan, J.-H.; Brody, S.L. Sex hormone-dependent regulation of cilia beat frequency in airway epithelium. Am. J. Respir. Cell Mol. Biol. 2012, 46, 446-453. [CrossRef]

51. Modelska, K.; Cummings, S. Tibolone for postmenopausal women: Systematic review of randomized trials. J. Clin. Endocrinol. Metab. 2002, 87, 16-23. [CrossRef]

52. Cho, M.K.; Park, H.M. The National Use of Hormonal Therapy in Postmenopausal Women in 2010. J. Korean Soc. Menopause 2011, 17, 150-154. [CrossRef]

53. Ferri, S.; Crimi, C.; Heffler, E.; Campisi, R.; Noto, A.; Crimi, N. Vitamin D and disease severity in bronchiectasis. Respir. Med. 2019, 148, 1-5. [CrossRef] [PubMed] 